\title{
Loa loa
}

National Cancer Institute

\section{Source}

National Cancer Institute. Loa loa. NCI Thesaurus. Code C123512.

A species of parasitic nematodes in the family Onchocercidae. The lifecycle of L. loa involves deer flies as the intermediate host and humans as the definitive host. Infestation causes loa loa filariasis (loiasis). 